Proceedings of the 2012 Winter Simulation Conference

C. Laroque, J. Himmelspach, R. Pasupathy, O. Rose, and A.M. Uhrmacher, eds

\title{
JOINT STAFF J7 ENVIRONMENT DEVELOPMENT SUPPORT FOR NATO SIMULATION ACTIVITIES
}

\author{
Amy Grom \\ Joint Staff J7 \\ 116 Lakeview Parkway \\ Suffolk, Virginia, USA 23435
}

\author{
Andy Bowers \\ The MITRE Corporation \\ 903 Enterprise Parkway Suite 200 \\ Hampton, Virginia, USA 23666
}

\begin{abstract}
The Environment Development Branch (ED), United States Joint Staff J7 (JS J7), formerly the Joint Forces Command (JFCOM) J7, supports a variety of simulation environment development activities in concert with NATO and Partnership for Peace (PfP) countries. Recent activities include development of the NATO Training Federation (NTF) in support of an experiment running in parallel with the "traditional" Southeastern Europe Simulation (SEESIM) training exercise, support for NATO Research \& Technology Organization (RTO) Task Group MSG-106, Enhanced CAX Architecture, Design and Methodology, and development of NTF to support NATO Joint Force training. This paper introduces each of these activities, discusses synergies achieved among them, and addresses the influence JS J7 participation in NATO/PfP activities has had on ED development activities in support of US Joint Force training.
\end{abstract}

\section{INTRODUCTION}

The United States Joint Staff J7 (JS J7), formerly the Joint Forces Command (JFCOM) J7, fulfills its coalition responsibilities in numerous ways, including supporting coalition exercises, sponsoring observer/trainer teams in support of coalition partners, supporting the Afghanistan Mission Network, and supporting a variety of simulation environment development activities in concert with coalition countries (Moore 2011). This paper focuses on the last of these efforts, specifically a selection of simulation development activities to improve training for NATO and partner forces. These activities include JS J7 Environment Development (ED) support for NATO Research \& Technology Organization (RTO) Task Group MSG-106, development of the NATO Training Federation (NTF) in support of an experiment running in parallel with the "traditional" Southeastern Europe Simulation (SEESIM) training exercise, and development of NTF to support NATO Joint Force training. ED support of these three activities: MSG-106, SEESIM 12, and NTF development, are discussed respectively in sections 2, 3, and 4. Section 5 reports on the influence these activities have had on ED development of the Joint, Live, Virtual, and Constructive (JLVC) federation in support of US Joint Force training. We present conclusions in Section 6.

ED supports the three activities because they are conducted collaboratively with coalition partners, because they support US Joint Force training, and because they are complementary. With respect to the latter, the large number of simulations and simulators developed over the years to support a variety of training needs eventually led to a community dedicated to enabling interoperability among these systems. The three activities, four including JLVC, addressed in this paper are fundamentally simulation interoperability efforts. Moreover, the standards used to enable interoperability in each activity are those associated with the High Level Architecture (HLA), more specifically IEEE Std $1516^{\mathrm{TM}}-2010$, commonly called HLA Evolved. This paper presumes familiarity with the HLA and so the authors freely use the language of HLA without definition or explanation. Readers unfamiliar with these terms are referred to the standard (IEEE 2010). 


\section{Grom and Bowers}

Table 1 provides the authors' view of the synergy present among the four activities. The first column includes numbers subsequently used for reference. They are not intended to indicate priority. The "Features" column lists different features which the activities support to various degrees. The activities appear in the column headings of the four right-most columns. Cell entries in the four right-most columns contain the author's evaluation of the relationship of the features to the respective activities. The scale used is 1 to 5 with 5 being primary importance and 1 being minimal importance.

Table 1 does not, however, capture the temporal aspect of how development in support of one activity subsequently supports another activity. For example, SEESIM appears to have the least in common with the other efforts described in the paper, but this is due to the short development period for SEESIM and the relatively large development step undertaken to move from a single Federation Description Document (FDD) comprising the Real-time Platform Reference FOM (RPR FOM) version 2 draft 17 (v2d17) used during previous MSG-068 work to a set of new RPR FOM modules which will be subsequently used by MSG-106, NTF, and JLVC Evolved. Notwithstanding this deficiency, the table may be useful in visualizing how the activities relate to each other as each is presented in its respective section. Throughout the paper, references to table number rows appear in curly brackets. For example, $\{\# 1\}$ refers to an activity related to "explicit scenario initialization strategy."

Table 1: EDB HLA Evolved-Related Development

\begin{tabular}{|c|c|c|c|c|c|}
\hline \# & Features & $\begin{array}{c}\text { MSG- } \\
106\end{array}$ & NTF & SEESIM & $\begin{array}{c}\text { JLVC } \\
\text { "Evolved" }\end{array}$ \\
\hline 1 & Explicit scenario initialization strategy & 5 & 5 & & 5 \\
\hline 2 & Scenario scalability (support for large entity counts) & 5 & 3 & & 5 \\
\hline 3 & $\begin{array}{l}\text { Support for diverse federate update rate requirements (i.e. } \\
\text { L vs V vs C) }\end{array}$ & 5 & 4 & & 5 \\
\hline 4 & $\begin{array}{l}\text { Support causality among select federate(s) for select trans- } \\
\text { actions }\end{array}$ & & 5 & & 5 \\
\hline 5 & $\begin{array}{l}\text { Support allocation of modeling responsibility to different } \\
\text { federates for a given object }\end{array}$ & 5 & 5 & & 3 \\
\hline 6 & $\begin{array}{l}\text { Support dynamic transfer of modeling responsibility } \\
\text { among federates }\end{array}$ & 5 & 5 & & 3 \\
\hline 7 & Support modular object modeling (modular FOMs) & 5 & 5 & 5 & 5 \\
\hline 8 & Support for select NETN_FOM modules (w/modification) & 5 & 4 & 4 & 4 \\
\hline 9 & Support for unreliable federates & 5 & 5 & & 5 \\
\hline 10 & Improved C2-Simulation interoperability & 5 & & & 4 \\
\hline
\end{tabular}

\section{ED SUPPORT FOR MODELING AND SIMULATION GROUP (MSG)-106}

MSG-106, Enhanced CAX Architecture, Design and Methodology, met for the first time in February 2012. The MSG was initiated in response to recommendations by MSG-068, NATO Education and Training Network (NETN), notably those for additional technical development and improved assessment of operational support requirements (MSG-068 2011). ED is co-chairing the MSG-106 Operational Subgroup to focus on the latter as a means of improving training for NATO and partner forces. ED is also cochairing the MSG-106 Technical Subgroup to address the specific recommendations from MSG-068 (MSG-106 2012) and to conduct other technical activities enabling improved training. This paper reports primarily on the technical activities ED is supporting within the MSG-106 Technical Subgroup rather than the Operational or Governance Subgroups' activities. The MSG is scheduled to work together for three years and since the MSG started in February 2012, the activities described below are at this point planned rather than completed. Thus the authors can neither report on accomplishments within each activ- 
ity or, based on lessons learned from MSG-068, guarantee that work will actually occur on activities scheduled for years two or three in the MSG's tenure.

The US contributions to MSG-106 include the Joint Conflict and Tactical Simulation (JCATS) and the Joint Theater Level Simulation (JTLS) because ED is the proponent for both simulations and can therefore direct development in both to support MSG-106 objectives. Moreover, since JCATS and JTLS were both used by the US to support MSG-068, work for MSG-106 will leverage work done in support of MSG-068. The developers for both simulations played an important role in MSG-068 by coding their respective simulations to support the FOM modules developed by MSG members thereby, together with simulations developed by other nations, providing a means to test the sufficiency of the FOM modules and associated Federation Agreements Documents (FADs).

\subsection{Correcting Shortfalls In The NETN Version 1 Federation Object Model (FOM)}

The NETN v1 FOM, one of the key deliverables of MSG-068, comprised several FOM modules including NETN Service Consumer-Provider, NETN Logistics, NETN Aggregate Unit, and the aforementioned RPR FOM.

- The primary technical shortfall noted in the MSG-068 final report regarding FOM modules developed by the group was that some of the modules were unnecessarily large and could be decomposed into more modular elements. For example, the NETN Aggregate Unit module included provisions for both multi-resolution modeling and a combat adjudication service. By the end of MSG-068 development, these capabilities were considered separable. Similarly, the Simulation Interoperability Standards Organization RPR FOM Product Development Group (PDG) is reviewing a set of FOM modules equivalent to the RPR FOM v2d17. The MSG-106 technical subgroup will therefore restructure the NETN Aggregate Unit module as five smaller modules: one apiece for the multi-resolution modeling and combat adjudication service and the remaining three leveraging select RPR FOM modules. $\{\# 7, \# 8\}$

- Other FOM updates, from naming conventions, to the revision of particular datatypes, to the introduction of a new datatype for a universally unique identifier (UUID) should improve the maintainability of FOM modules over time, thereby decreasing cost. For example, the value of uniquely identifying object instance has led to different implementations in different federations, to include the use of the "Marking" field in the RPR FOM and both Callsign and UniqueID in NETN. Several studies have recommended a more formal use of unique identifiers (Bowers and Gregg 2010). Introducing a UUID will help to resolve the inconsistencies in implementation while avoiding the ambiguity which results from overloading terms such as Callsign.

\subsection{IEEE Std $1516^{\mathrm{TM}}-2010$ Features}

The MSG-068 final report noted that, with the exception of FOM modularity, the MSG had little time to experiment with features of the new HLA specification, IEEE Std $1516^{\mathrm{TM}}-2010$. The report recommended future development and testing of Smart Update Rate Reduction (SURR), Data Distribution Management (DDM), and Fault Tolerance (MSG-068 2011). MSG-106 intends on working with these features. Though technical in nature, they promise to improve training for NATO and partner forces as described below. 


\section{Grom and Bowers}

- SURR enables systems with different tolerances for update rates to co-exist in a HLA simulation environment (Moller and Karlsson 2005). This potentially improves training both by increasing the variety of system types, that is Live versus Virtual versus Constructive systems, which can be used together in a simulation environment to produce training stimuli and by increasing the "scalability" of simulation environment, that is, enabling increased numbers of simulated objects. As training audiences have increasingly been challenged with operations in urban areas or operations within range of non-combatants, simulation providers have sought to enrich the simulation environment by increasing the number of non-combatants, non-combatant vehicles, noncombatant electronic emissions, and so on. $\{\# 2, \# 3\}$

- The MSG-106 technical sub-group also intends on working with DDM as it complements SURR in improving scalability. DDM allows federates to publish object attributes and interactions to specific "dimensions." Subscribing federates may select dimensions of interest and the RunTime Infrastructure (RTI) only delivers data to those dimensions. $\{\# 2, \# 3\}$

- Fault Tolerance encompasses a variety of techniques (Moller et al. 2005) for improving the reliability of the simulation environment. This has become increasingly important as C2-simulation interoperability has increased and disruptions in the simulation environment are more noticeable to training audiences as tracks disappear from C2 systems or data becomes stale when not updated by an inoperable federate. $\{\# 9\}$

\subsection{C2-Simulation Interoperability}

The MSG-068 final report also recommended that a follow-on activity develop and test a pattern(s) for C2-simulation interoperability. MSG-085 Coalition Battle Management Language (CBML) has made extensive progress on, and contributed to, SISO's work on CBML standards (Khimeche et al. 2011). MSG106 intends on leveraging the work accomplished by MSG-085 and SISO and extending it by developing a FOM module(s) which supports CBML transactions. There are, at present, two proposals within MSG106 for the structure of the module. The first proposes that the CBML transaction consists of two components, the first providing meta-data about the message and the second containing the message itself. The second proposal recommends that the CBML transaction includes as many components as the message type requires; that is, the different fields comprising the CBML message are each components of the FOM transaction. $\{\# 10\}$

\subsection{Scenario Initialization}

The MSG-068 final report recommended that a follow-on activity implement a scenario initialization process to combat problems with uncorrelated data. MSG-106 again intends on leveraging work accomplished by MSG-085 and SISO. In an effort complementing CBML, MSG-085 worked with scenario initialization and the SISO standard for Master Scenario Definition Language (MSDL) (Pullen et al. 2012). $\{\# 1\}$

\subsection{Transfer Of Modeling Responsibility}

The MSG-068 final report recommended that a pattern(s) for Transfer of Modeling Responsibility (TMR) be developed by a follow-on activity (MSG-068 2011). In addition to adding flexibility to the modeling environment by allowing transfer of an object from control of one federate to control by another, TMR allows different federates to own different attributes of a single object. For example, a simulator might update the spatial attribute of an aircraft object specifying its location, velocity, and direction of flight, while the damage state attribute might be updated by a physics-based damage simulation. This promises to improve training audience stimuli because representation is being done by the most appropriate simulation. $\{\# 6\}$ 


\section{ED SUPPORT OF SEESIM TRAINING EXERCISE}

The Southeastern Europe Simulation (SEESIM) exercise has occurred every two years since 2002 to promote regional cooperation, coordination and interoperability among the South-Eastern Europe Defense Ministerial (SEDM) nations. The scenarios have typically featured natural calamities, earthquakes for example, with resultant human suffering whose alleviation requires crisis management response by a variety of national agencies. JS J7 will continue the JFCOM practice of supporting SEESIM using JTLS. As in previous years, a cadre of personnel from JS J7 will transport the simulation to the theatre, operate it during the exercise, and remove it at the end of the exercise. SEESIM 12 will again rely on JTLS, but this year an experiment will run in parallel with the "traditional" exercise. The genesis of this experiment lies in the Bulgarian desire to establish a persistent CAX support infrastructure in the region using National simulations. Since several of the countries in the region use JCATS and some use JTLS to support National training, a federation of JTLS and multiple instances of JCATS would provide the simulation components of the CAX infrastructure. This federation already exists; JTLS and JCATS comprise the two principle federates in the NATO Training Federation (NTF), discussed in further detail in section 4. Bulgaria, the SEESIM 12 host, proposed using JCATS to support tactical-level National training and JTLS to represent operational-level forces and equipment, and entities outside of Bulgarian control.

While SEESIM 12 planners stopped short of embracing the Bulgarian proposal and direct NTF support of the SEESIM exercise, stakeholders agreed to conduct SEESIM 12 in tandem with an experiment using NTF. Should the experiment prove successful, future exercises in the series might use the NTF architecture. An unexpected benefit of this compromise is the involvement of HQ Supreme Allied Commander Transformation (HQ SACT) Operational Experimentation Branch (OPEX) to formally evaluate the use of NTF and other NATO resources. Two other nations, Croatia and Serbia, both of which own JCATS, will participate with Bulgaria in the experiment by representing their national assets in JCATS in support of their National training objectives and relying on JTLS as an "umbrella" federate representing forces and equipment not controlled by Croatia, Serbia, or Bulgaria.

OPEX's principle objective is to "assess the value of having NATO ... participate in a non-NATO led multinational exercise, such as SEESIM12, from the perspective of NATO and participating nations." With respect to NTF, OPEX's objective is to "assess the viability and utility of using the NATO Training Federation (NTF), ... in a non-NATO led multi-national exercise"(White 2012) The OPEX team authored an Experiment Design Document complete with Measures of Effectiveness, Measures of Performance, and a data collection plan. In addition to answering their charter, OPEX's report promises an unusually complete picture of the infrastructure and resources necessary to support a simulation environment supporting a regional exercise. This is of obvious interest to the MSG-106 Operational Subgroup, while the MSG-106 Technical Subgroup members are interested in NTF development and execution details.

As mentioned above, a short development period and the decision to use the new RPR FOM modules as the basis for the SEESIM NTF interoperability constrained the development team to enabling relatively few federation transactions $\{\# 7, \# 8\}$. Bulgarian JCATS users may be limited to observing JTLS aircraft, vehicles, and so on owned by other nations or agencies outside of their control, and the JCATS equipment owned by Croatia or Serbia. This may prove sufficient for the purposes of the experiment as National training audiences observe the results of their cooperation and coordination when, for example, assets owned by one nation arrive at locations agreed upon with others.

\section{NATO TRAINING FEDERATION (NTF)}

The NTF, introduced above, evolved from the JFCOM J7-developed Joint Multi-Resolution Model (JMRM) federation. NATO's Joint Warfare Centre used the NTF to support exercise STEADFAST JOINER 2008 (SJ08) and thus it predates both MSG-106 and SEESIM 12. Since SJ08, however, NTF has undergone substantial transformation, adopting the HLA Evolved standard and the NETN FOM during the MSG-068 development years. Shortly after MSG-068 concluded in 2011, NATO convened a NTF

Configuration Control Board (CCB) which approved eleven developmental requirements, six of which 


\section{Grom and Bowers}

are reflected by entries in Table 1. The CCB identified a "User Need" and an associated "Change Requirement." User needs encompassed scenario-related issues such as Joint Personnel Recovery or Time Sensitive Targeting, as well as federation management issues such as cost and reliability. The CCB requirements were then prioritized from 1, highest, to 11, lowest. CCB-established requirements are summarized below in priority order, with the exception that different requirements with the same technical solution are combined (NTF CCB 2011).

- Priority 1. Compliance with new standards and recommendations by the nations and, more specifically, compliance with IEEE Std $1516^{\mathrm{TM}}-2010$ and NETN. As it is consistent with US requirements for JTLS and JCATS, ED has initiated work on this CCB requirement. $\{\# 7, \# 8\}$

- Priority 2. This requirement specifies the need for an effective and efficient scenario initialization strategy. A related requirement of lesser priority addresses adding objects dynamically during runtime. $\{\# 1\}$

- Priority 3. Robustness and reliability. This requirement encompasses a fault tolerance strategy in case of a federate or federation crash. $\{\# 9\}$

- Priority 4. A capability to operate NTF faster than real-time, twice real time being minimally acceptable and 2.5 times real time as an objective. This requirement implies use of HLA timemanagement services and while time-management is not an explicit activity identified in Table 1, it would simplify implementing items 4,5 , and 6. $\{\# 4, \# 5, \# 6\}$

- Priority 5. Use of Virtual Battlespace 2 (VBS2) and streaming video to support targeting and intelligence training objectives. VBS2, and virtual simulations more generally, are typically constrained in the number of objects which they can process during subscription. Therefore, some means of regulating the data sent to VBS2 is needed. $\{\# 2, \# 3\}$

- Priority 6. Reliable ownership transfer and provable causality. $\{\# 4, \# 5, \# 6\}$

- Priority 7. Practical and efficient federation management to include backups, starting and terminating federation, recovering from a crash, checkpoints, federation speed. This requirement is not explicitly addressed in Table 1. MSG-106 activities include federation management control, but the US has not committed to supporting this activity, currently scheduled to start in the first quarter 2013.

\section{INFLUENCE ON ED DEVELOPMENT IN SUPPORT OF US JOINT FORCE TRAINING}

ED participation in simulation development activities to improve training for NATO and partner forces has affected development in support of US Joint Force training. JS J7 leadership's decision in January 2012 to migrate the Joint Live, Virtual, and Constructive (JLVC) federation, the US flagship Joint simulation environment, to the IEEE Std $1516^{\mathrm{TM}}-2010$ specification was in part based on ED personnel participation in MSG-068 and their experience with the specification's modular FOM feature $\{7\}$. The JLVC, a federation of US Service, Joint, and Agency simulations and systems, has grown significantly since its inception in support of exercise Millennium Challenge 2002 (MC02). Integration costs have likewise grown as regression testing includes federates regardless of whether they were directly involved in implementing new functionality. JS J7 leadership anticipates cost savings using FOM modules since federates not involved in a functional upgrade need not participate in integration testing and subsequent use of the associated module. JS J7 will also realize opportunity cost savings as these same federates will have additional time to develop other functions or features.

As ED implemented JS J7 leadership's decision, the JCATS developers work accomplished in support of SEESIM 12 enabled JLVC management use of JCATS to test the JLVC version 6.0 FOM prior to releasing it to the larger federation. The JCATS developers assisted in resolving problems in twelve versions of the FOM prior to its release to the other federate developers. As the FOM matured with use by the larger federation, modules developed for SEESIM were reused in the JLVC version 6.0 FOM. In short, ED participation in SEESIM 12 has influenced JLVC development. $\{7,8\}$ 


\section{Grom and Bowers}

Initial work in support of MSG-106 also promises change in JLVC. For example, ED participants in the MSG-106 Technical Subgroup circulated the proposed DDM scheme to members of the JLVC community. The ensuing JLVC community discussion recommended changes to the proposal, but more importantly from the JLVC standpoint, recommended significant changes in JLVC's DDM implementation. These include replacing the current dimensions with ones aligned with the MSG-106 proposal and shifting the burden of multicast assignments from the federates to the RTI. This promises to simplify federate code, thereby reducing the difficulty and cost of changes while promoting interoperability with NATO or partner federates. $\{2\}$ ED will participate in the C2-Simulation interoperability activity within MSG-106 for the expressed purpose of evaluating a capability not currently used in JLVC. $\{10\}$ ED will lead the MSG-106 work on a combat adjudication service, currently scheduled to begin in 2013, in large part to learn lessons of import to JLVC which utilizes multiple combat adjudication simulations.

ED work with the IEEE Std $1516^{\mathrm{TM}}-2010$ specification in concert with MSG-106, NTF, and to a lesser extent, SEESIM, may benefit future JLVC development as ED can assess features not currently used in JLVC at relatively small cost in comparison to testing their implementation in JLVC. For example, ED might evaluate SURR during the course of MSG-106 or fault tolerance in NTF as a precursor to using these features in the full JLVC. $\{2,3,9\}$

Further in the future, modular FOMs developed in support of MSG-068, MSG-106, NTF, or JLVC promise to support transition to the "next generation" JLVC, JLVC-2020, comprised of cloud-enabled modular M\&S services. JLVC-2020 seeks to capitalize on the same features that make FOM modules so useful, that is, flexibility, accuracy, and reusability, by modularizing M\&S services. Reuse promises dramatic cost reduction across the defense department since particular objects, and even methods on objects, are currently duplicated in multiple simulations. Decomposing monolithic simulations into "best of class" modules and reusing these promise significant cost reduction and improved capability. Coincidently, "rehosting" these services in the cloud broadens the reuse opportunities, as it exposes simulation use outside of traditional simulation centers.

\section{CONCLUSION}

ED participates in a variety of activities in support of NATO and coalition partners. This paper has reported on a subset of these activities involving current simulation development to improve training for NATO and partner forces: MSG-106, SEESIM 12, and NTF. ED supports these activities as part of its commitment to coalition engagement and to exploit the synergies among these activities and current or anticipated ED development activities in support of US Joint Force training. All of these current activities utilize the IEEE Std $1516^{\mathrm{TM}}-2010$ as they fundamentally require interoperability between or among diverse simulations. Though only US simulations are named in this paper, the FOM modules and HLA features developed and tested in support of the activities promote interoperability between US, NATO, and coalition partner simulations. As ED designs the future JLVC-2020, it recognizes that linkages to current partner simulations will remain important and indicates as much in "to be" architectures.

\section{REFERENCES}

Bowers, F., and B. Gregg. 2010. "Use of Unique Identifiers to Enable Interoperability Among LVC Components." In Proceedings of the 2010 NATO Modeling and Simulation Conference, Utrecht, Netherlands.

IEEE (2010) IEEE $1516^{\mathrm{TM}}$-2010 IEEE Standard for Modeling and Simulation (M\&S) High Level Architecture (HLA)-Framework and Rules.

Khimeche, L., et al. 2011. "C2 \& Simulation Coupling - Lessons Learned from German \& French CBML Experimentation." Proceedings of the 2011 Fall Simulation Interoperability Workshop, Orlando, Florida. Accessed July 16. http://www.sisostds.org/DigitalLibrary.aspx?Command=Core_Download\&EntryId=34913. 
Moller, B., and M. Karlsson. 2005. "Developing well-balanced federations using the HLA Evolved Smart Update Rate Reduction." Proceedings of the 2005 Fall Simulation Interoperability Workshop, Orlando, Florida. Accessed July 16. http://www.sisostds.org/DigitalLibrary.aspx?Command=Core Download\&EntryId=26459.

Moller, B., et al. 2005. "Developing Fault Tolerant Federations using HLA Evolved." Proceedings of the 2005 Spring Simulation Interoperability Workshop, San Diego, California. Accessed July 16. http://www.sisostds.org/DigitalLibrary.aspx?Command=Core_Download\&EntryId=26664.

Moore, J. 2011. "Solutions for the Coalition Training Environment." In Proceedings of the 2011 International Training and Education Conference, Cologne, Germany.

MSG-068 2011. NATO Education and Training Network (NETN) Final Technical Report. Accessed July 16. http://www.cso.nato.int/Pubs/rdp.asp?RDP=RTO-TR-MSG-068.

MSG-106 2012. Enhanced CAX Architecture, Design and Methodology. Background and Objectives. Accessed July 16. http://www.cso.nato.int/ACTIVITY_META.asp?ACT=MSG-106.

NATO Training Federation Configuration Control Board Minutes September 162011.

Pullen, M., et al. 2012. "MSDL and C-BML Working Together for NATO MSG-085." Proceedings of the 2012 Spring Simulation Interoperability Workshop, Orlando, Florida. Accessed July 16. http://www.sisostds.org/DigitalLibrary.aspx?Command=Core Download\&EntryId=35464.

White, E. 2012. "DTE Experimentation in SEESIM 12” Main Planning Conference Plenary Brief 3 April 2012.

\section{AUTHOR BIOGRAPHIES}

AMY GROM is a US government civilian and the Coalition Engagement Officer within Joint Staff J, Synchronization and Integration Division, Joint Operating System Environment, Environment Development Branch. She is the US representative to NATO Modeling and Simulation Group 106 and serves as the technical sub-group co-chair. She is also the Action Officer for the ACT SEESIM NTF Experiment. As an additional duty, she is the Foreign Military Sales coordinator for joint simulation software. Ms. Grom has a Bachelor degree in Computer Science from Western Connecticut State University. Her email address is amy.m.grom.civ@mail.mil.

ANDY BOWERS is a Lead Simulation Modeling Engineer in the MITRE Corporation's Modeling \& Simulation Engineering Department. He is a retired United States Army officer and has more than 15 years of experience in simulation and federation design and development for military training. He has supported several NATO Modeling and Simulation Group (NMSG) efforts and currently supports the Joint Live, Virtual, Constructive (JLVC) Training Federation System Engineering Team. His interests include multi-resolution modeling and simulation interoperability. Mr. Bowers has a Bachelor of Science degree from the United States Military Academy and a Master of Science in Operations Research Systems Analysis and a Master of Science in Civil Engineering from Stanford University. His email address is bowersa@mitre.org. 